\title{
Wet Spinning and Drawing of Human Recombinant Collagen
}

\section{Supporting Information}

\author{
Amit Yaari $^{1}$, Yaelle Schilt ${ }^{2}$, Carmen Tamburu ${ }^{2}$, Uri Raviv ${ }^{2}$ and Oded Shoseyov ${ }^{1,3} *$
}

1 The Robert H. Smith Faculty of Agriculture, Food and Environment, and the Center for

Nanoscience and Nanotechnology, the Hebrew University of Jerusalem, P.O.B. 12, Israel

2 Institute of Chemistry and the Center for Nanoscience and Nanotechnology, the

Hebrew University of Jerusalem, Jerusalem 91904, Israel

3 CollPlant Ltd. 3 Sapir Street, POB 4132, Israel

Corresponding Author:

*Phone: +972-54-2341863 Mail: Shoseyov@agri.huji.ac.i1

This supporting information section contains 5 pages, 4 figures and 1 table. 


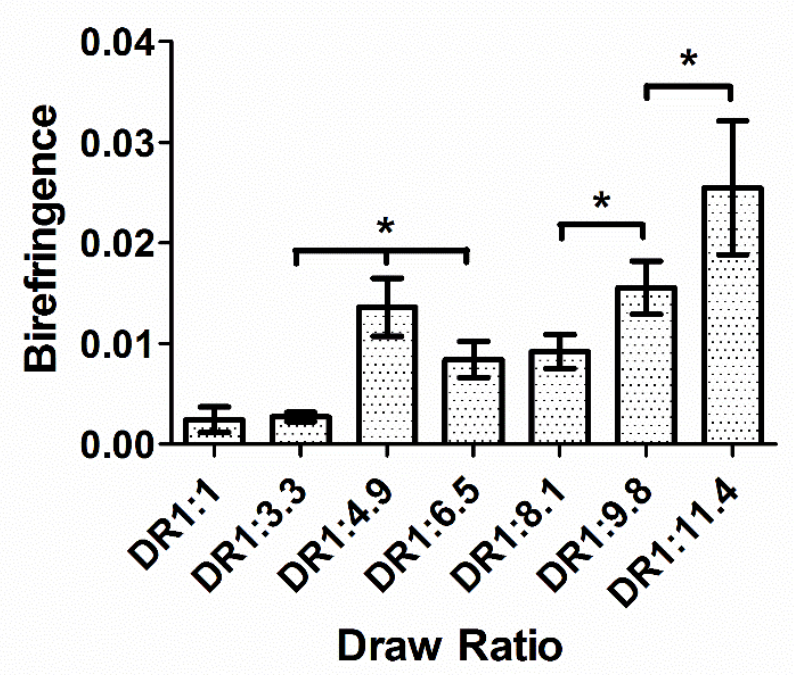

Figure S1: A plot of the birefringence versus draw ratio. The birefringence was calculated by dividing the retardance by the fiber diameter. Error bars shows the standard deviation (SD). $\mathrm{n}=5,6,6,6,7,6,5$, for DR1 to 11.4 , respectively. Asterisk denotes statistical significance of $p<0.05$. For birefringence calculation, the diameter (D) of the fiber and retardance value (R) were measured at the same point along the fiber. Birefringence for every fiber sample was then calculated as the average R/D of 5-7 individual measurements, as previously described ${ }^{1}$. 


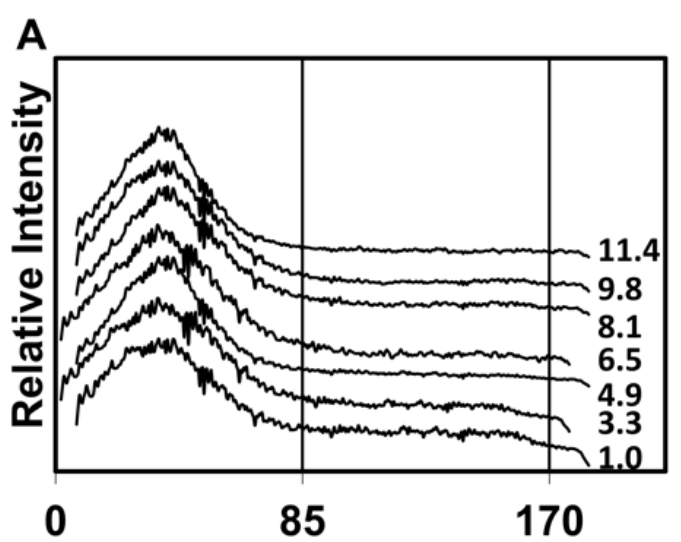

[Deg]

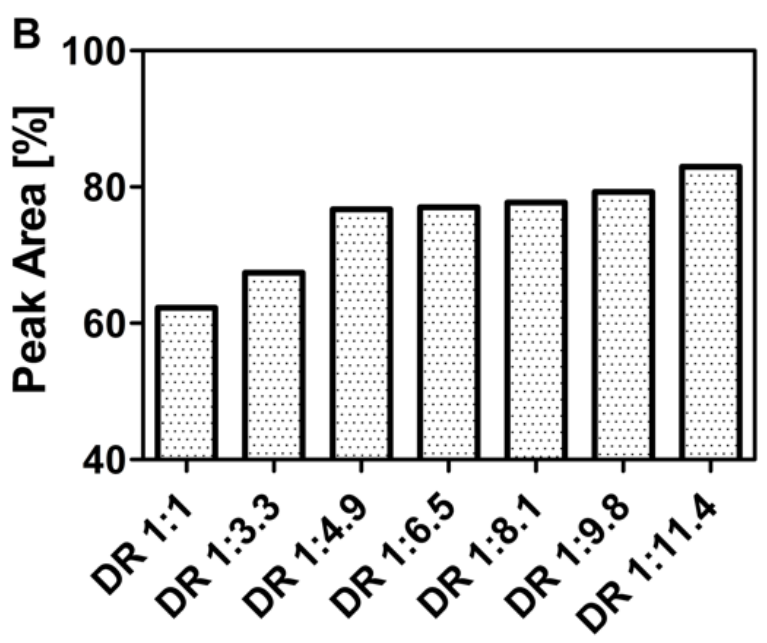

Draw Ratio

Figure S2: Small angle X-ray scattering analysis. A - Azimuthal integrations of the diffraction profiles obtained from fibers drawn under different DR conditions. B - Ratio of the area under the peak (from $0^{\circ}$ to $85^{\circ}$ ) to the total area of the signal (from $0^{\circ}$ to $180^{\circ}$ ). It can be seen that the ordered area relative to the total signal area gradually increased with the draw ratio, reflecting the increased order of the collagen monomers in the fiber.

High-resolution SAXS measurements were performed in the previously described setup 2. The axes of the fibers were positioned in the plain normal to the X-ray beam. In this plane, all the fibers were positioned at the same angle to the vertical axis. After dark current and air scattering subtraction, azimuthal integration of the 2D scattering image was carried out using the FIT2D software developed at the European Synchrotron Radiation Facility (ESRF) ${ }^{3}$. The ratio of oriented to nonoriented material was calculated as follows: for each sample, the peak area (from $0^{\circ}$ to $85^{\circ}$ ) of the azimuthal scan was divided by the total signal area from $0^{\circ}$ to $180^{\circ}$ (normalized for material quantity and exposure). 
A

Dry Extension at Break

Wet Extension at Break
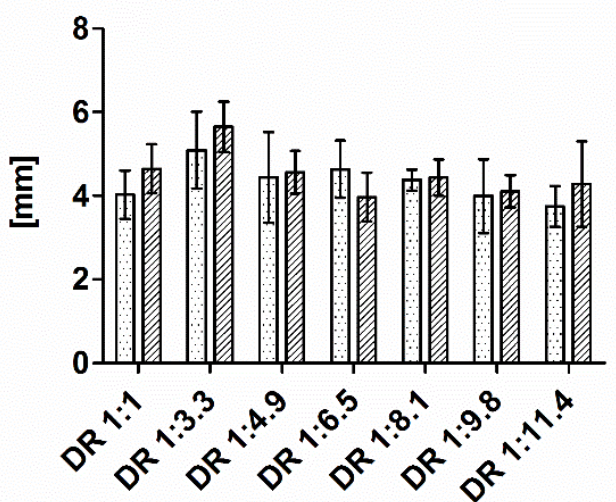

Draw Ratio

C

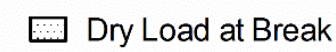

Wet Load at Break

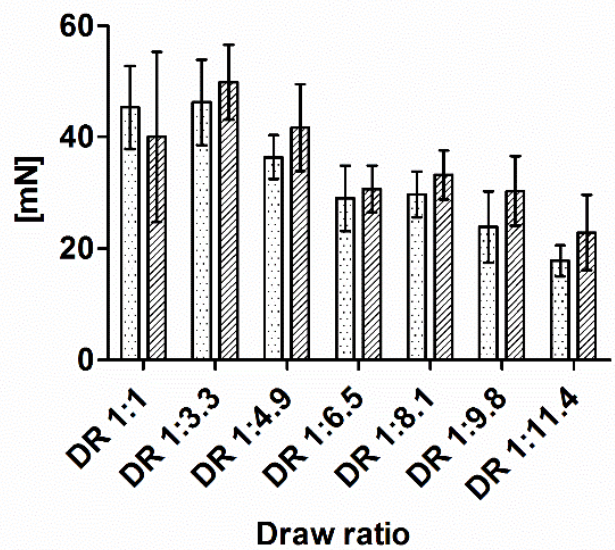

B

Dry Tensile stress at Break

Wet Tensile stress at Break

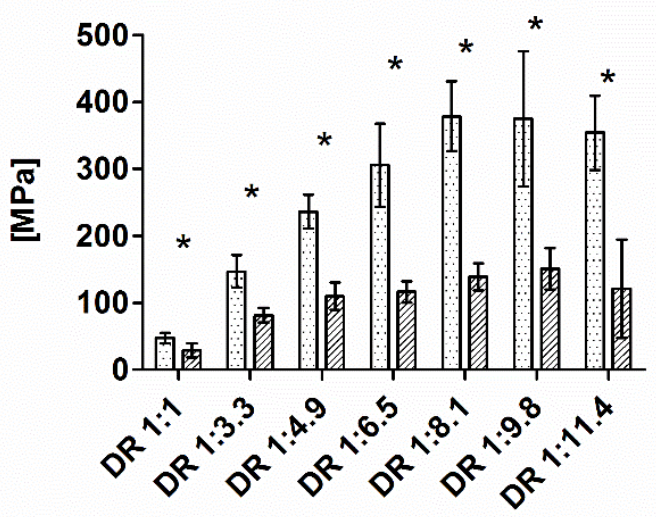

Draw Ratio
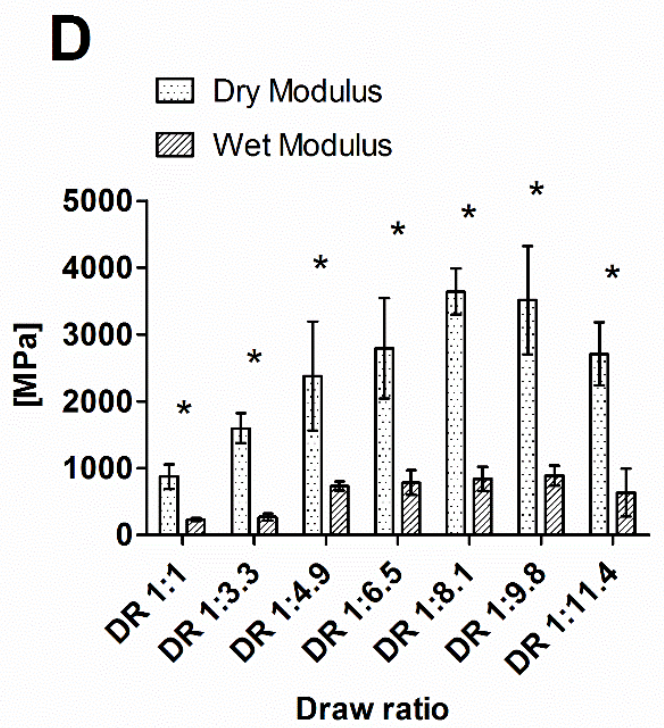

Figure S3: A comparison of mechanical properties between dry and wet rhcollagen fibers. A - Extension at break. B - Stress at break. C - Load at break. D - Young's modulus. Error bars show the SD. Asterisk denotes statistical significance of $\mathrm{p}<0.05$. 
A

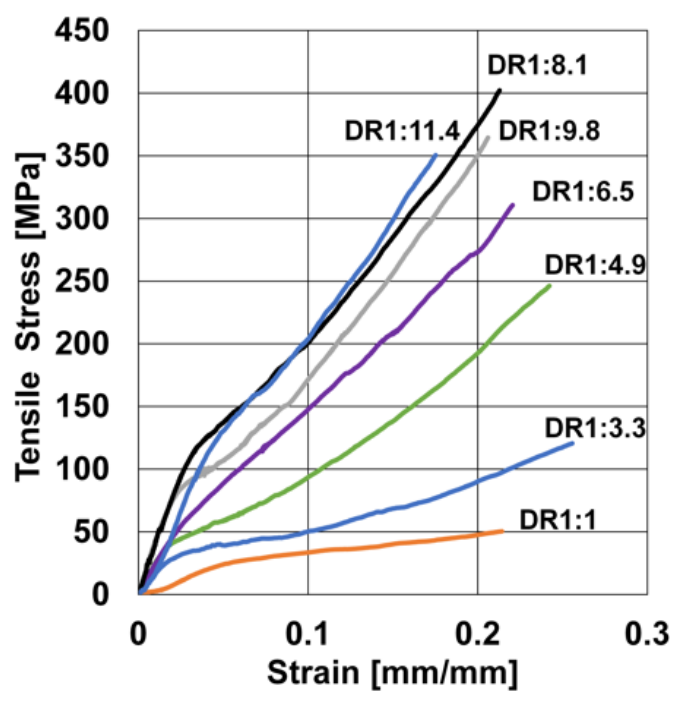

B

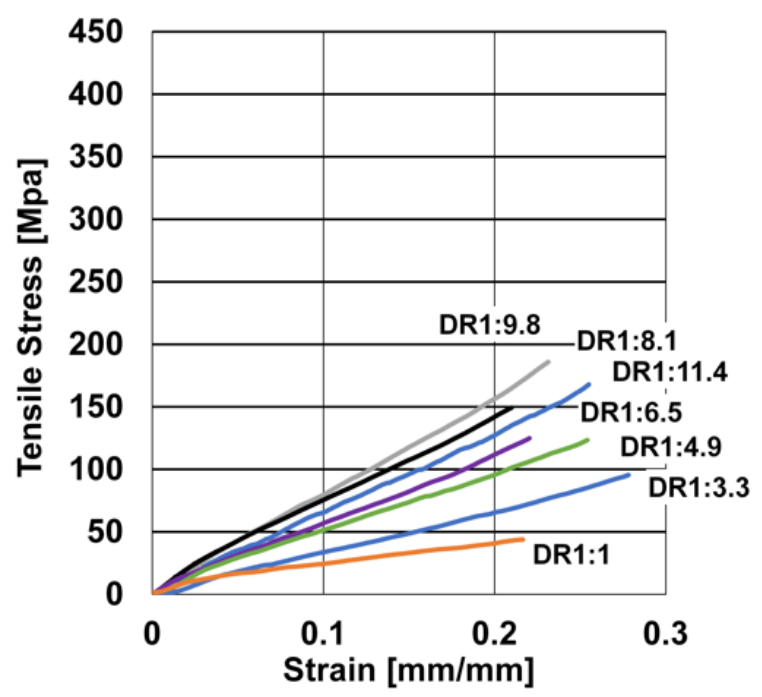

Figure S4: Representative stress strain plots of fibers spun at different draw ratios. A-

Dry fibers, B- hydrated fibers.

Table S1: fiber dimensions and spinning rate.

$\begin{array}{cccc}\text { Draw Ratio } & \begin{array}{l}\text { Dry Diameter } \\ {[\mu \mathrm{m}]}\end{array} & \begin{array}{l}\text { Length per mg } \\ {[\mathrm{m}]}\end{array} & \begin{array}{l}\text { Spinning r } \\ {[\mathrm{m} / \mathrm{h}]}\end{array} \\ \text { DR1:1 } & 35 \pm 3.1 & 1.7 \pm 0.1 & 91 \\ \text { DR1:3.3 } & 20 \pm 2.7 & 5.6 \pm 0.3 & 304 \\ \text { DR1:4.9 } & 14 \pm 2.3 & 8.5 \pm 0.4 & 456 \\ \text { DR1:6.5 } & 11 \pm 1.3 & 11.3 \pm 0.6 & 608 \\ \text { DR1:8.1 } & 10 \pm 2.5 & 14.1 \pm 0.7 & 761 \\ \text { DR1:9.8 } & 9 \pm 1.3 & 16.9 \pm 0.8 & 913 \\ \text { DR1:11.4 } & 8 \pm 0.8 & 19.7 \pm 1.0 & 1,065\end{array}$




\section{References:}

1. Um, I. C.; Ki, C. S.; Kweon, H.; Lee, K. G.; Ihm, D. W.; Park, Y. H., Wet spinning of silk polymer: II. Effect of drawing on the structural characteristics and properties of filament. International journal of biological macromolecules 2004, 34 (1), 107-119.

2. Nadler, M.; Steiner, A.; Dvir, T.; Szekely, O.; Szekely, P.; Ginsburg, A.; Asor, R.; Resh, R.; Tamburu, C.; Peres, M., Following the structural changes during zinc-induced crystallization of charged membranes using time-resolved solution X-ray scattering. Soft Matter 2011, 7 (4), 1512-1523.

3. Huang, T.; Toraya, H.; Blanton, T.; Wu, Y., X-ray powder diffraction analysis of silver behenate, a possible low-angle diffraction standard. Journal of applied crystallography 1993, 26 (2), 180-184. 